\title{
Negative thermal expansion and antiferromagnetism in the actinide oxypnictide $\mathrm{NpFeAsO}$
}

\author{
T. Klimczuk, ${ }^{1,2,{ }^{*}}$ H. C. Walker, ${ }^{3,4}$ R. Springell, ${ }^{5,6}$ A. B. Shick, ${ }^{1,7}$ A. H. Hill,,${ }^{3,}$ P. Gaczyński, ${ }^{1}$ K. Gofryk, ${ }^{8}$ S. A. J. Kimber, ${ }^{3}$ \\ C. Ritter, ${ }^{9}$ E. Colineau, ${ }^{1}$ J.-C. Griveau, ${ }^{1}$ D. Bouëxière, ${ }^{1}$ R. Eloirdi, ${ }^{1}$ R. J. Cava, ${ }^{10}$ and R. Caciuffo ${ }^{1}$ \\ ${ }^{1}$ Institute for Transuranium Elements, JRC, European Commission, Postfach 2340, 76125 Karlsruhe, Germany \\ ${ }^{2}$ Faculty of Applied Physics and Mathematics, Gdansk University of Technology, Narutowicza 11/12, 80-952 Gdansk, Poland \\ ${ }^{3}$ European Synchrotron Radiation Facility, 6 rue Jules Horowitz, Boîte Postale 220, 38043 Grenoble Cedex 9, France \\ ${ }^{4}$ Deutsches Elektronen-Synchrotron DESY, 22607 Hamburg, Germany \\ ${ }^{5}$ London Centre for Nanotechnology and Department of Physics and Astronomy, University College London, \\ London WC1E 6BT, United Kingdom \\ ${ }^{6}$ Royal Commission for the Exhibition of 1851 Research Fellow, Interface Analysis Centre, University of Bristol, \\ Bristol BS2 8BS, United Kingdom \\ ${ }^{7}$ Institute of Physics, ASCR, Na Slovance 2, 18221 Prague 8, Czech Republic \\ ${ }^{8}$ Los Alamos National Laboratory, Los Alamos, New Mexico 87545, USA \\ ${ }^{9}$ Institute Laue-Langevin, 6 rue Jules Horowitz, Boîte Postale 156, 38042 Grenoble Cedex 9, France \\ ${ }^{10}$ Department of Chemistry, Princeton University, Princeton, New Jersey 08544, USA \\ (Received 5 April 2012; revised manuscript received 20 April 2012; published 9 May 2012)
}

\begin{abstract}
A neptunium analog of the $\mathrm{LaFeAsO}$ tetragonal layered compound has been synthesized and characterized by a variety of experimental techniques. The occurrence of long-range magnetic order below a critical temperature $T_{\mathrm{N}}=57 \mathrm{~K}$ is suggested by anomalies in the temperature-dependent magnetic susceptibility, electrical resistivity, Hall coefficient, and specific-heat curves. Below $T_{\mathrm{N}}$, powder neutron diffraction measurements reveal an antiferromagnetic structure of the $\mathrm{Np}$ sublattice, with an ordered magnetic moment of $1.70 \pm 0.07 \mu_{B}$ aligned along the crystallographic $c$ axis. No magnetic order has been observed on the Fe sublattice, setting an upper limit of about $0.3 \mu_{B}$ for the ordered magnetic moment on the iron. High-resolution x-ray powder diffraction measurements exclude the occurrence of lattice transformations down to $5 \mathrm{~K}$, in sharp contrast to the observation of a tetragonal-to-orthorhombic distortion in the rare-earth analogs, which has been associated with the stabilization of a spin-density wave on the iron sublattice. Instead, a significant expansion of the $\mathrm{NpFeAsO}$ lattice parameters is observed with decreasing temperature below $T_{\mathrm{N}}$, corresponding to a relative volume change of about $0.2 \%$ and to an Invar behavior between 5 and $20 \mathrm{~K}$. First-principles electronic structure calculations based on the local spin density plus Coulomb interaction and the local density plus Hubbard-I approximations provide results in good agreement with the experimental findings.
\end{abstract}

DOI: 10.1103/PhysRevB.85.174506

PACS number(s): 75.50.Ee, 74.70.Xa, 65.40.De, 61.05.fm

\section{INTRODUCTION}

Rarely in physics has a single family of compounds generated as much interest as the iron pnictide superconductors. ${ }^{1-5}$ Research in this field is being conducted at such a hectic pace that no fewer than 2500 articles have cited the paper that reported the discovery of superconductivity in $\mathrm{LaFeAsO}_{1-x} \mathrm{~F}_{x}{ }^{6}{ }^{6}$ The reason for this frenzy of activity is clear: the superconducting transition temperatures are very high, second only to the cuprates.

The replacement of the rare-earth $(R)$ species in the $R \mathrm{FeAsO}$ "1111" phase with $\mathrm{Np}$, without changing the roomtemperature structure, and the ability to subsequently replace $\mathrm{Np}$ with heavier actinides $(\mathrm{Pu}$ and $\mathrm{Am})$ represent a real possibility to control correlations (from weak to strong, $\mathrm{Np}$ to Am) and to potentially extend the already rich physics of the iron pnictides.

The parent $R \mathrm{FeAsO} 1111$ compounds have so far involved rare-earth $3^{+}$ions in the oxide layers. These have ionic radii $\sim 1 \AA$ and have partially filled $4 f$ shells, which are atomic-like in nature. The influence of the oxide layer in the behavior of these materials, beyond being a "spacer," is a seldom-addressed question. In fact, from a materials point of view this is a difficult problem to investigate since elements that possess similar chemical properties to the rare earths, both in terms of bonding and size, are hard to find. For ideal candidates one needs to descend to the bottom of the periodic table, specifically, the transuranic actinide elements, $\mathrm{Np}, \mathrm{Pu}$, and Am. These have ionic radii of almost identical magnitude to the majority of the lanthanides and are commonly present in the trivalent state in similar compounds. Not only do they represent an alternative to the lanthanides per se, but they can also be used to study the effect of the degree of localization of the $f$ electron states (in this case $5 f$ ) on the observed properties. This is a commonly adopted strategy in the actinide community when investigating new compounds; the behavior of the actinide metals as one traverses the period from uranium to americium changes from itinerant, transition-metal-like electronic behavior to localized, atomic-like behavior.

Here, we report on the synthesis and characterization of $\mathrm{NpFeAsO}$, an isostructural $\mathrm{Np}$ analog of $\mathrm{LaFeAsO}$. Magnetic, electrical transport, and specific-heat measurements have been used to characterize its macroscopic physical properties, and neutron and $\mathrm{x}$-ray diffraction have been used to monitor the evolution of the magnetic and crystallographic structures with decreasing temperature. The experiments show the development of an antiferromagnetic order on the $\mathrm{Np}$ sublattice below $T_{\mathrm{N}}=57 \mathrm{~K}$ and an absence of observable crystallographic distortions down to $5 \mathrm{~K}$. This is at variance with the behavior of the rare-earth analogs $R \mathrm{FeAsO}$, where the occurrence of a 
tetragonal-to-orthorhombic lattice transformation is observed well above the critical temperature of the rare-earth sublattice. On the other hand, the magnetic transition in $\mathrm{NpFeAsO}$ is accompanied by a large expansion of the unit cell volume below $T_{\mathrm{N}}$ followed by an Invar behavior below about $20 \mathrm{~K}$. First-principles calculations of the electronic structure provide a Fermi surface with almost two-dimensional character, very similar to the one previously reported for $R \mathrm{FeAsO}$, and predict an antiferromagnetic ground state with a staggered magnetization along the tetragonal $c$ axis. The anomalous thermal expansion is qualitatively described within a localdensity plus Hubbard-I approximation.

\section{EXPERIMENT}

The first and not inconsiderable hurdle one has to overcome when studying these materials is sample synthesis. This is nontrivial in the case of the radioactive and toxic actinides. One point of caution at this stage is that we have started our study with $\mathrm{Np}$, whereas it is most common to begin with U. For the case of the oxypnictides, replacing the rare-earth element by $U$ is highly unlikely to be successful, given that $U$ can be either tri- or tetravalent and is the latter in the vast majority of oxygen-containing U-based compounds.

Polycrystalline samples of $\mathrm{NpFeAsO}$ were synthesized at the Institute for Transuranium Elements (ITU) by solid-state reaction using $\mathrm{Fe}_{3} \mathrm{O}_{4}$ (Alfa Aesar 99.997\%), elemental Fe (Alfa Aesar 99.998\%), and crystals of NpAs as starting materials. The thoroughly mixed powder was pressed into a pellet, sealed in an evacuated silica ampoule, and heated at $900{ }^{\circ} \mathrm{C}$ for $48 \mathrm{~h}$, before finally being furnace quenched. All operations were carried out in a radioprotected glove box with low oxygen and water concentrations.

High-resolution $\mathrm{x}$-ray diffraction data were measured as a function of temperature $(5 \leqslant T \leqslant 300 \mathrm{~K})$ on the powder diffraction beam line, ID31, at the European Synchrotron Radiation Facility (ESRF), France, using an incident beam energy of $35 \mathrm{keV}$. The powdered sample for this experiment was doubly encapsulated because it was put inside a Kapton tube $(50 \mu \mathrm{m})$ and inserted in a Plexiglas hollow cylinder with a wall thickness of $200 \mu \mathrm{m}$.

The magnetic susceptibility measurements were performed at ITU on a Quantum Design MPMS-7 superconducting quantum interference device (SQUID) magnetometer in applied magnetic fields $\mu_{0} H_{1}=5 \mathrm{~T}$ and $\mu_{0} H_{2}=7 \mathrm{~T}$, where we define $\chi_{D C}=\left[M\left(H_{2}\right)-M\left(H_{1}\right)\right] /\left(H_{2}-H_{1}\right)$. Electrical resistivity, the Hall effect, and heat capacity were measured using a Quantum Design Physical Properties Measurement System (PPMS-9). The electrical resistivity was determined using a standard four-probe dc technique, with four 0.05 -mm-diameter platinum wires glued to the sample using silver epoxy (Epotek $\mathrm{H} 20 \mathrm{E}$ ). The Hall resistance $R_{H}$ was determined by voltage measurements $V_{H}$ under applied magnetic field at +9 and $-9 \mathrm{~T}$. The field response $V_{H}(B)$ at fixed temperatures has been measured to confirm results obtained when ramping in temperature. The heat capacity was measured using a standard relaxation calorimetry method, where the data were corrected for the contribution of the Stycast encapsulation material (2850 FT) by using an empirical relation determined previously.
Neutron powder diffraction was performed on the highintensity two-axis D20 diffractometer at the Institut Laue Langevin (ILL), France, in the high-flux mode, with an incident wavelength of $2.42 \AA$, using a (002) pyrolitic graphite monochromator. About $250 \mathrm{mg}$ of $\mathrm{NpFeAsO}$ powder was used within a triple-walled Al container. Data were collected at 5, 30, 90, 190, and $290 \mathrm{~K}$ using the ILL transuranic sample orange cryostat. Variable temperature ramp data were also collected between 30 and $90 \mathrm{~K}$.

\section{RESULTS AND DISCUSSION}

Upon the successful synthesis of $\mathrm{NpFeAsO}$, our initial step was to check the crystal structure and sample purity using $\mathrm{x}$-ray powder diffraction. At room temperature this indicates that this $\mathrm{Np}$ compound is an isostructural analog to the celebrated rare-earth iron oxypnictide family $R \mathrm{FeAsO}$, as demonstrated by the successful refinement of the high-resolution data shown in Fig. 1 to the $\mathrm{ZrCuSiAs}$-type structure. Extra lines appearing in the diffraction pattern correspond to $\mathrm{NpO}_{2}$, which is the only observable impurity phase (about $8 \%$ in weight). On cooling to $5 \mathrm{~K}$ no orthorhombic distortion was observed within the resolution of our data [inset (a)], in contrast to all of the $R \mathrm{FeAsO}$ compounds [such as $\mathrm{SmFeAsO}$ in inset (b)]. The absence of the crystallographic distortion immediately prompts a question regarding the nature of the magnetism in $\mathrm{NpFeAsO}$ since it is believed that in the rare-earth compounds the distortion is intrinsically related to the development of a spin-density wave on the iron, breaking the rotational symmetry within the plane. Inspection of Fig. 2 reveals that bulk thermodynamic magnetic and transport property measurements give no indication of an anomaly within the standard temperature range for the $R F$ FeAsO spin-density wave ordering (120-140 K). Instead, anomalies are only observed at $25 \mathrm{~K}$, corresponding to the phase transition in the $\mathrm{NpO}_{2}$ impurity phase, ${ }^{8}$ and significantly at $\simeq 60 \mathrm{~K}$.

Even more striking and unusual, however, is the observed variation of the lattice parameters as a function of temperature. As shown in Figs. 1(c) and 1(d), the standard thermal expansion behavior stops abruptly below $T \sim 60 \mathrm{~K}$, and as the temperature is decreased, the crystallographic cell expands. The very accurate data obtained at the ESRF ID31 high-resolution powder diffraction beam line allows us to study the effect along both principal crystallographic directions in detail. Although the temperature dependences for both $a$ and $c$ lattice parameters show clear minima, $T_{\min }$, the $c$-axis minimum is about $10 \mathrm{~K}$ higher than in the $a$-axis direction. Below $T_{\min }$ the $c$ lattice parameter increases monotonically and saturates for $T<20 \mathrm{~K}$. On the contrary, the $a$ lattice parameter reaches a maximum at around $30 \mathrm{~K}$ and then starts to decrease with decreasing temperature. The relative lattice size change differs by a factor of 4 , with the one in the $c$-axis direction being greater. The above conclusions are not affected by the presence of the $\mathrm{NpO}_{2}$ impurity, whose lattice parameter shows a regular temperature behavior with a contraction at the onset of electric quadrupole order $\left(T_{0}=25 \mathrm{~K}\right) .^{9}$

Using the second-order Grüneisen approximation for the zero-pressure equation of state, ${ }^{10,11}$ the volumetric data were 

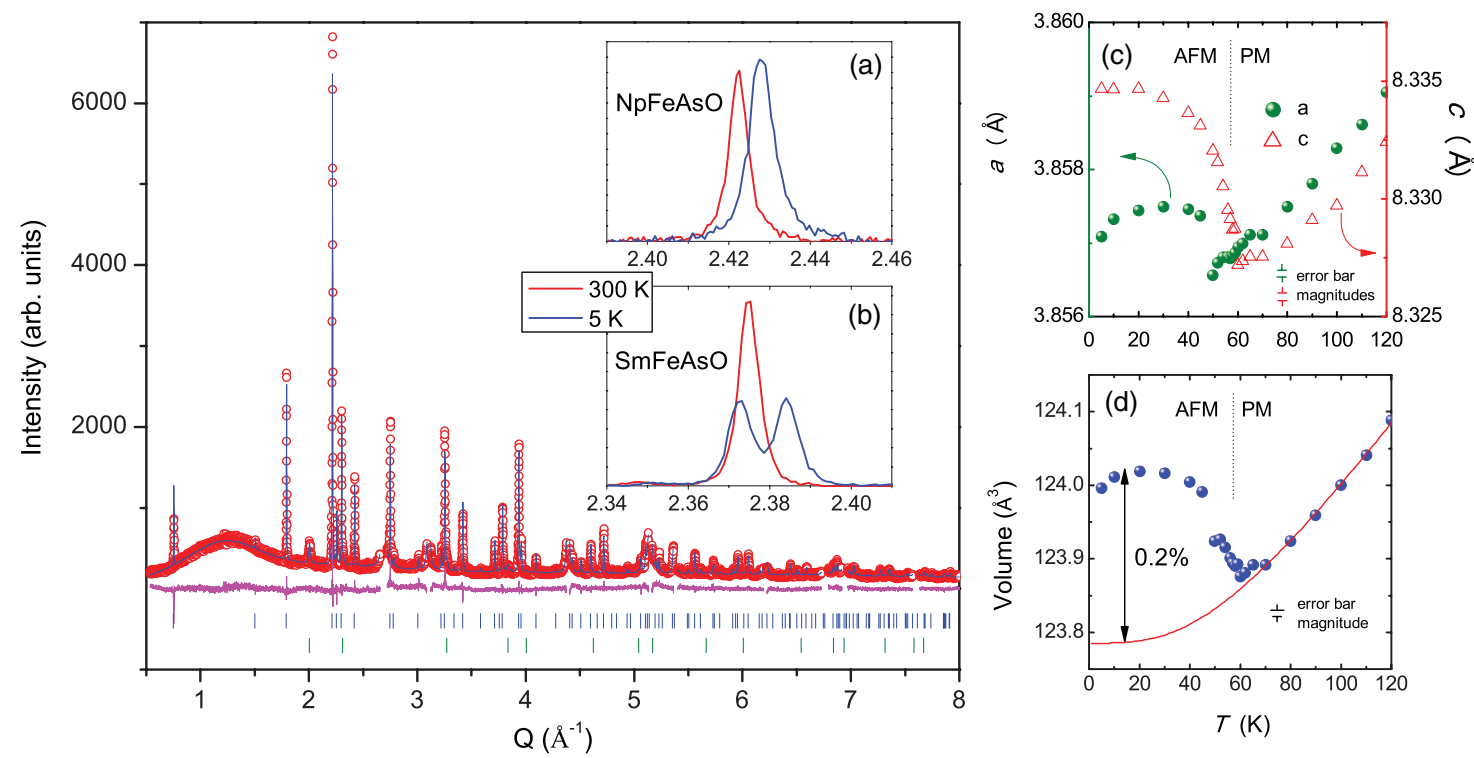

FIG. 1. (Color online) Fit (solid blue line) of the refined structural model (Rietveld method) to the high-resolution room-temperature x-ray powder diffraction data (red circles) for $\mathrm{NpFeAsO}$, confirming the tetragonal $\mathrm{P} 4 / \mathrm{nmm} \mathrm{ZrCuSiAs}$-type structure. The data were collected on the ID31 (ESRF) high-resolution x-ray powder diffractometer with a wavelength of $\lambda=0.35 \AA$. Tick marks are shown for two refined phases: $\mathrm{NpFeAsO}$ (top, blue) and an $8 \% \mathrm{NpO}_{2}$ impurity (bottom, green). Insets (a) and (b) make a comparison of data for $\mathrm{NpFeAsO}$ and $\mathrm{SmFeAsO}{ }^{7}$ where only $\mathrm{SmFeAsO}$ shows the splitting of the room-temperature (red line) tetragonal $(\mathrm{P} 4 / \mathrm{nmm})(111)$ Bragg reflection into the orthorhombic $(\mathrm{Cmma})(201)$ and (021) reflections at $5 \mathrm{~K}$ (blue line). Insets (c) and (d) show the variation in the $a$ and $c$ lattice parameters and of the unit cell volume as a function of temperature, as extracted from the $\mathrm{x}$-ray powder diffraction measurements, revealing a marked negative thermal expansion below $T \simeq 60 \mathrm{~K}$ in $\mathrm{NpFeAsO}$. The red line through the volume data is a fit to the second order of the Grüneisen approximation for the zero-pressure equation of state, as discussed in the text.

modeled according to

$$
V(T)=\frac{V_{0} U}{Q-b U}+V_{0},
$$

where $U$ is the internal energy, which we have calculated following the Debye approximation,

$$
U(T)=9 N k_{B} T\left(\frac{T}{\Theta_{D}}\right)^{3} \int_{0}^{\Theta_{D} / T} \frac{x^{3} d x}{e^{x}-1},
$$

where $N$ is the number of atoms in the unit cell, $k_{B}$ is the Boltzmann constant, and $\Theta_{D}$ is the Debye temperature, leading to $\Theta_{D}=287 \mathrm{~K}, Q=0.52 \mathrm{eV}, b=260$, and $V_{0}=123.78 \AA^{3}$ and hence a negative thermal expansion of $\omega=\Delta V / V=$ $0.2 \%$.

The presence and form of the anomaly in the magnetic susceptibility [Fig. 2(a)] at $\sim 57 \mathrm{~K}$ imply the onset of antiferromagnetic ordering and therefore that the anomalous thermal expansion is a consequence of a strong coupling between the magnetism and the lattice. In order to estimate the precise value of the Néel temperature, we followed Ref. 12 and plotted $d(\chi T) / d T$ (solid line) on the same temperature scale. The maximum of $d(\chi T) / d T$ is observed at $T_{\mathrm{N}}=57 \mathrm{~K}$. Above $\sim 100 \mathrm{~K}$, the susceptibility curve has a Curie-Weiss behavior, with an effective paramagnetic moment $\mu_{\text {eff }}=2.78 \pm 0.06 \mu_{\mathrm{B}}$ and a Curie-Weiss temperature $\Theta_{\mathrm{CW}}=$ $-128 \pm 2 \mathrm{~K}$. The above values have been obtained by fitting the inverse susceptibility curve to a straight line, taking into account the contribution of the $\mathrm{NpO}_{2}$ impurity phase by scaling the data reported in Ref. 13.
In the intermediate coupling scheme, $\mu_{\text {eff }}$ of $\mathrm{Np}^{3+}$ is $2.755 \mu_{\mathrm{B}}$, and hence our results imply that the neptunium is trivalent, as is the case for the rare-earth species in the $R \mathrm{FeAsO}$ isostructural compounds. The magnetic moment on the iron is likely to be small given that the majority of the magnetism can be attributed to the $\mathrm{Np}$ moments. The negative CurieWeiss temperature suggests the presence of antiferromagnetic interactions. An additional anomaly is present in the data at $T=25 \mathrm{~K}$. This corresponds to the magnetic triakontadipole phase transition of $\mathrm{NpO}_{2},{ }^{14-16}$ which is present as an impurity phase in our sample, as detected in the $\mathrm{x}$-ray diffraction pattern.

Figure 2(b) shows the resistivity, revealing a sharp drop at the magnetic transition. The low-temperature resistivity can be described by the presence of antiferromagnetic (AFM) interactions at high temperatures with the opening of a magnetic gap near the magnetic transition temperature, ${ }^{17}$ as demonstrated by the fit in the inset to the function (blue solid line):

$$
\rho(T)=\rho_{0}+a T^{2}+b T(1+2 T / \Delta) \exp ^{-\Delta / T},
$$

where $\rho_{0}=70 \pm 1 \mu \Omega \mathrm{cm}, a=0.27 \pm 0.01 \mu \Omega \mathrm{cm} \mathrm{K}^{-2}, b=$ $20 \pm 1 \mu \Omega \mathrm{cm} \mathrm{K}^{-1}$, and $\Delta=49 \pm 1 \mathrm{~K}$. The value of $\Delta$ is comparable to that obtained for $\mathrm{NpCoGa}_{5}(\Delta=55 \mathrm{~K})$, which orders antiferromagnetically at $T_{\mathrm{N}}=47 \mathrm{~K} .{ }^{18}$

While the electrical resistivity indicates that $\mathrm{NpFeAsO}$ is a reasonable metal, the number and nature of the carriers were further investigated by means of the Hall effect. The temperature dependence of the Hall effect of $\mathrm{NpFeAsO}$ is 

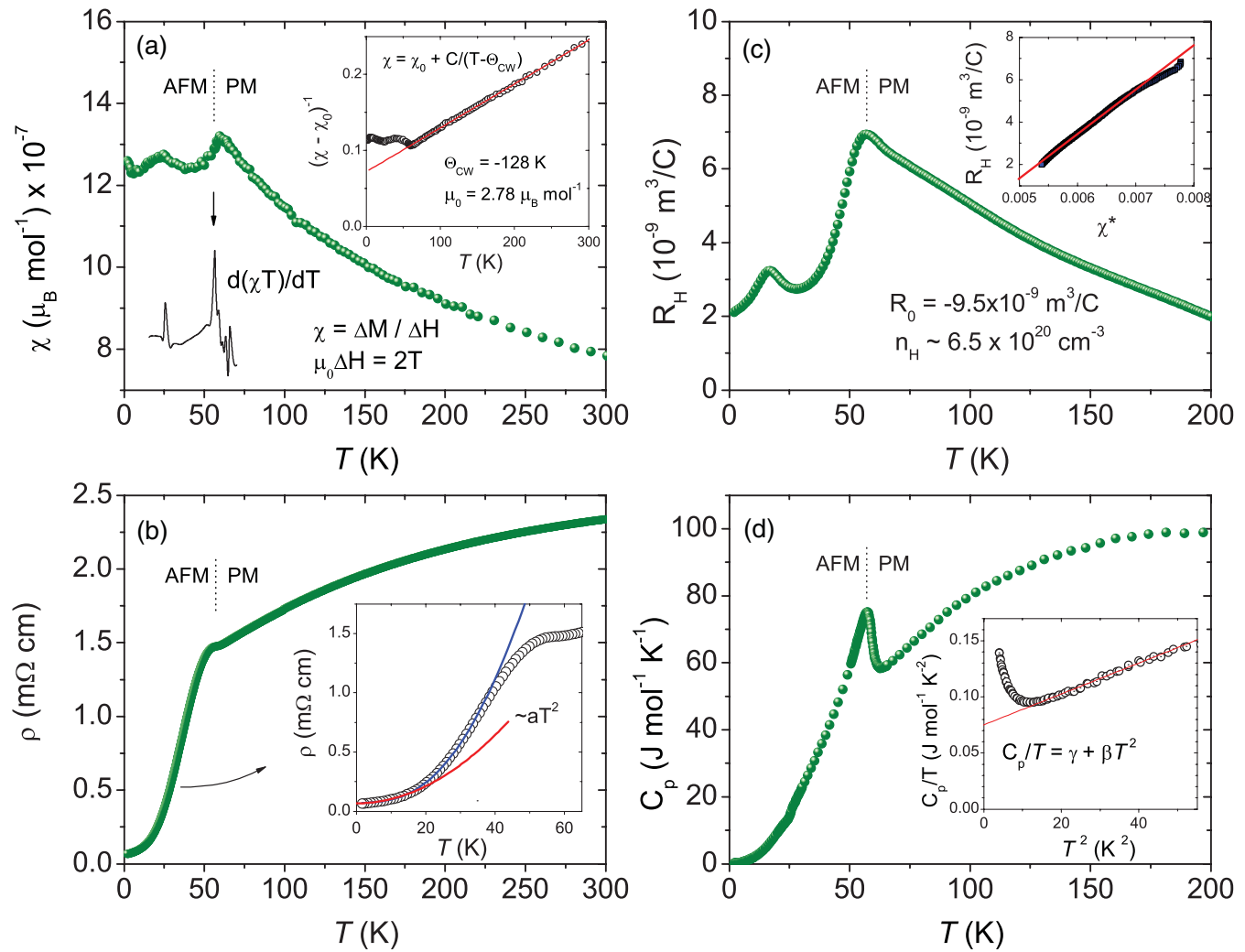

FIG. 2. (Color online) Bulk physical properties of NpFeAsO. (a) The magnetic susceptibility $\chi=\Delta M / \Delta H$ data (solid circles) and $d(\chi T) / d T$ (solid line). The inset shows a Curie-Weiss fit to the inverse susceptibility from which an effective paramagnetic moment of $2.78 \pm 0.06 \mu_{\mathrm{B}}$ and a Curie-Weiss temperature of $-128 \pm 2 \mathrm{~K}$ are obtained. (b) The electrical resistivity measured in $H=0 \mathrm{~T}$. The inset highlights the magnetically ordered region, showing two fits to the data, indicating that the low-temperature resistivity does not vary simply as $T^{2}$ (red line); instead, an improved fit is obtained after including an antiferromagnetic gap (blue line). (c) The Hall coefficient as a function of temperature. The inset shows the least-squares fit described in the text. (d) The heat capacity, including an inset showing a fit to the low-temperature data.

shown in Fig. 2(c). At room temperature the Hall coefficient $R_{H}$ is positive and of the order of $2 \times 10^{-9} \mathrm{~m}^{3} \mathrm{C}^{-1}$. It increases with decreasing temperature down to the Néel temperature $T_{\mathrm{N}}=57 \mathrm{~K}$, where $R_{H}(T)$ exhibits a distinct maximum, reminiscent of the maximum in the temperature dependence of the magnetic susceptibility [see Fig. 2(a)]. It has been shown experimentally and theoretically, based on the Anderson periodic model with the crystal electric field effect, that in magnetic materials the Hall coefficient may be described as the sum of two parts:

$$
R_{H}(T)=R_{0}+R_{s} \chi^{*}(T),
$$

where $R_{0}$ is the normal Hall effect due to the Lorenz motion of carriers and the second term, the anomalous Hall effect, is related to the magnetic scattering. ${ }^{19-21}$ The reduced susceptibility $\chi^{*}(T)$ is approximated by $\chi(T) / C$ where $C$ is the Curie-Weiss constant. ${ }^{20}$ In the case of $\mathrm{NpFeAsO}$, a least-squares fitting of the above equation to the experimental data in the temperature range $60-200 \mathrm{~K}$ [see the inset in Fig. 2(c)] resulted in the values $R_{0}=-9.5 \times 10^{-9} \mathrm{~m}^{3} \mathrm{C}^{-1}$ and $R_{s}=2.1 \times 10^{-6} \mathrm{~m}^{3} \mathrm{C}^{-1}$ for the normal and anomalous Hall coefficients. The anomalous Hall effect, likely caused by the magnetism of the $\mathrm{Np}$ ions, is dominant, and therefore a positive $R_{H}$ is observed. However, the negative value of $R_{0}$ indicates that electrons are the dominant carriers, most probably due to their higher mobility. The single-band model provides an estimate for the concentration of free electrons of $6.5 \times 10^{20} \mathrm{~cm}^{-3}$, which should be considered as the upper limit of the actual carrier concentration in $\mathrm{NpFeAsO}$. Interestingly, the values of the ordinary Hall coefficient as well as its sign and the carrier concentration obtained for $\mathrm{NpFeAsO}$ are similar to the ones derived for similar $R \mathrm{FeAsO}$ systems based on the lanthanide elements. ${ }^{22-25}$ Neither resistivity nor Hall effect measurements on $\mathrm{NpFeAsO}$ show anomalies as observed around $150 \mathrm{~K}$ in transport property measurements of $R \mathrm{FeAsO}(R=\mathrm{La}, \mathrm{Ce}, \mathrm{Pr}, \mathrm{Nd}){ }^{23}$

Figure 2(d) presents the heat-capacity data measured down to $2 \mathrm{~K}$. Two anomalies are clearly identified, namely, a sharp $\lambda$-type cusp at $\sim 60 \mathrm{~K}$ that coincides with the onset of antiferromagnetic order in $\mathrm{NpFeAsO}$ and a shoulder at $25 \mathrm{~K}$, associated with the multipolar phase transition of the $\mathrm{NpO}_{2}$ impurity. ${ }^{26}$ The inset shows a fit to $C_{P} / T=\gamma+\beta T^{2}$ in the 3.5-7 K temperature range, where $\gamma T$ is the electronic contribution to the heat capacity and $\beta T^{3}\left(\beta=12 \pi^{4} N k_{B} / 5 \theta_{D}^{3}\right)$ is the acoustic phonon contribution in the low-temperature limit of the Debye model, where $\theta_{D}$ is the Debye temperature, $N$ is the number of atoms per formula unit, and $k_{B}$ is the Boltzmann constant. The contribution of the $\mathrm{NpO}_{2}$ impurity to the specific-heat data have been subtracted by scaling the data reported for $\mathrm{NpO}_{2}$ in Ref. 26. 
In the magnetically ordered state the magnetic specific-heat term $C_{\text {mag }}$ should be taken into account. In Ref. 18, the formula that describes the specific heat of magnons with an energy gap $\Delta$ in their dispersion relation, $C_{\mathrm{mag}}=\alpha T^{1 / 2} \exp (-\Delta / T)$, was successfully used to fit $C_{\mathrm{mag}}$ of $\mathrm{NpCoGa}_{5}$. Using the value of $\Delta=49 \mathrm{~K}$ obtained from the resistivity fit and taking $\alpha=3.7 \mathrm{~J}$ $\mathrm{mol}^{-1} \mathrm{~K}^{-3 / 2}$, as reported for $\mathrm{NpCoGa}_{5}$, the $C_{\text {mag }}$ term can be estimated. In the 3.5 to $7 \mathrm{~K}$ temperature range, $C_{\mathrm{mag}}$ changes from $6 \mu \mathrm{J} \mathrm{mol}^{-1} \mathrm{~K}^{-1}$ to $9 \mathrm{~mJ} \mathrm{~mol}^{-1} \mathrm{~K}^{-1}$, which are 5 and 2 orders of magnitude smaller than the measured value of $C_{P}$ and do not influence the fit shown in the inset of Fig. 2(d), where no deviations from linear behavior are observed. The values of $\gamma$ and $\Theta_{D}$ extracted from the fit are $75 \pm 1 \mathrm{~mJ} \mathrm{~mol}^{-1} \mathrm{~K}^{-2}$ and $178 \pm 2 \mathrm{~K}$, respectively. The obtained Debye temperature is not in good agreement with the value obtained from the fit of volumetric data. The observed upturn in $C_{P} / T$ below $T=3 \mathrm{~K}$ is caused by the nuclear Schottky anomaly, which is commonly observed in $\mathrm{Np}$ compounds. ${ }^{18,27}$ In order to calculate the magnetic entropy, the phonon contribution to the specific heat must be subtracted. However, we cannot use reported data for the parent $R \mathrm{FeAsO}$ because all of them undergo a structural phase transition such that at low temperature they posses the orthorhombic crystal structure. To our knowledge, despite of a lot of effort, synthesis of UFeAsO and ThFeAsO has not been successful. Therefore, the entropy removed upon magnetic ordering cannot be calculated at this time.

Given both $\gamma$ and $\chi(0 \mathrm{~K})$, which was obtained from the interpolation of the Curie-Weiss fit to $T=0 \mathrm{~K}$, the Wilson ratio,

$$
R_{W}=\frac{\pi^{2} k_{B}^{2}}{3 \mu_{\mathrm{eff}}^{2}}\left(\frac{\chi(0 K)}{\gamma}\right),
$$

can be estimated for $\mathrm{NpFeAsO}$, yielding a value for $R_{W}$ of 1.3. This is slightly higher than the free-electron value $\left(R_{W}=1\right)$ and less than expected for heavy-fermion compounds $\left(R_{W}=2\right)$.

Information about the magnetic structure was obtained from neutron powder diffraction. On cooling below $\sim 57 \mathrm{~K}$ additional Bragg reflections appeared (Fig. 3). Their absence

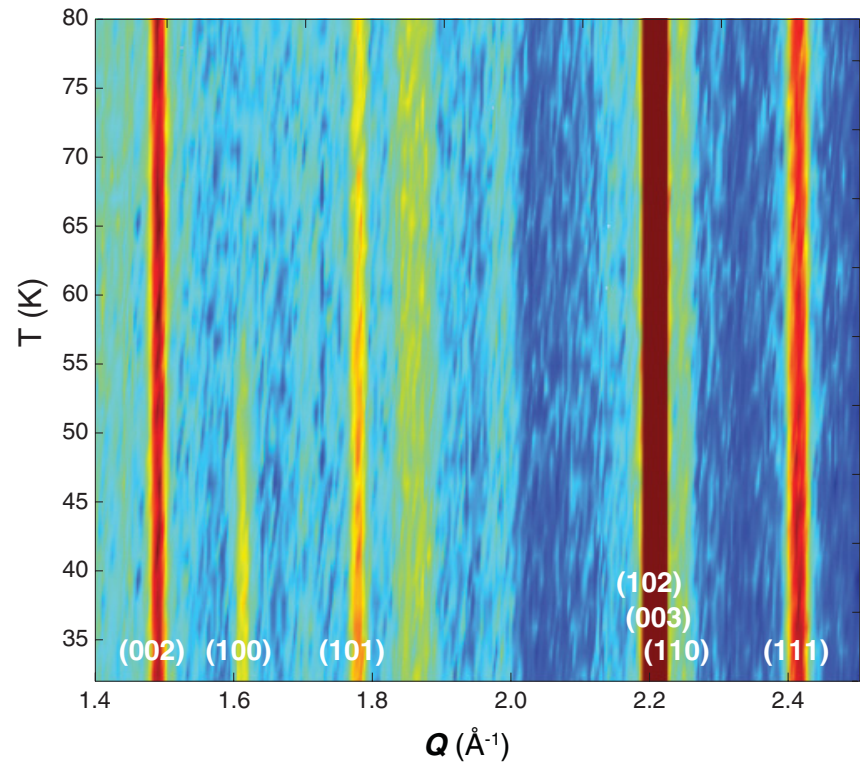

FIG. 3. (Color online) Variable-temperature neutron diffraction surface across $T_{\mathrm{N}}$, showing the appearance of the structurally forbidden (100) reflection and the increase in intensity of the (101) and (111) reflections due to magnetic ordering.

in the high-resolution x-ray powder diffraction data and their temperature dependence reflect their magnetic origin.

Rietveld modeling of the neutron data was performed using the GSAS program ${ }^{28}$ with EXPGUI. ${ }^{29}$ The magnetic form factor assuming spherical symmetry $\left(j_{0}\right.$ and $\left.j_{2}\right)$ and the bound coherent scattering length of ${ }^{237} \mathrm{~Np}^{3+}(10.55 \mathrm{fm})$ were taken from the international tables. ${ }^{30}$ A Landé splitting factor $g$ of 0.6 was used. Several parts of the diffraction patterns were excluded due to the aluminum container and other encapsulation and sample environment artifacts.

The 5 and $30 \mathrm{~K}$ data sets were fitted with the AFM $P 4 / n^{\prime} m^{\prime} m^{\prime}$ model, while the remaining sets were fitted with a paramagnetic $P 4 / \mathrm{nmm}$ model, the results of which are shown in Table I. To confirm that all the magnetic Bragg intensities were being modeled correctly, a purely magnetic

TABLE I. Crystallographic parameters as a function of temperature derived from Rietveld refinement of the structural model to match the neutron powder diffraction data from D20. Space group P4/nmm (origin choice 2) has been used (the ZrCuSiAs structure) with Np, Fe, As, and $\mathrm{O}$ on the $2 c, 2 b, 2 c$, and $2 a$ Wyckoff sites respectively. The Np magnetic moment $\left(\mathrm{Np} m_{z}\right)$ refined in the $P 4 / n^{\prime} m^{\prime} m^{\prime}$ magnetic space group in the low-temperature phase is also presented here.

\begin{tabular}{llllll}
\hline \hline & \multicolumn{5}{c}{$T(\mathrm{~K})$} \\
\cline { 2 - 5 } & \multicolumn{1}{c}{5} & \multicolumn{1}{c}{30} & \multicolumn{1}{c}{90} & \multicolumn{1}{c}{190} & \multicolumn{1}{c}{290} \\
\hline$a(\AA)$ & $3.85525(15)$ & $3.85512(15)$ & $3.85530(15)$ & $3.85851(16)$ & $3.86235(17)$ \\
$c(\AA)$ & $8.3374(6)$ & $8.3371(6)$ & $8.3324(6)$ & $8.3466(7)$ & $8.3663(7)$ \\
$\mathrm{Np} z$ & $0.1507(6)$ & $0.1508(6)$ & $0.1507(6)$ & $0.01512(7)$ & $0.1519(8)$ \\
$\mathrm{As} z$ & $0.6742(11)$ & $0.6742(10)$ & $0.6755(10)$ & $0.6749(10)$ & $0.6738(12)$ \\
$\mathrm{Np} U_{\text {iso }}\left(\AA^{2}\right)$ & $0.028(2)$ & $0.029(2)$ & $0.027(2)$ & $0.031(3)$ & $0.036(3)$ \\
$\mathrm{Fe} U_{\text {iso }}\left(\AA^{2}\right)$ & $0.032(3)$ & $0.032(3)$ & $0.033(3)$ & $0.034(3)$ & $0.034(3)$ \\
$\mathrm{As} U_{\text {iso }}\left(\AA^{2}\right)$ & $0.032(4)$ & $0.032(4)$ & $0.035(4)$ & $0.037(4)$ & $0.038(4)$ \\
$\mathrm{O} U_{\text {iso }}\left(\AA^{2}\right)$ & $0.028(5)$ & $0.029(5)$ & $0.027(4)$ & $0.033(5)$ & $0.039(5)$ \\
$\mathrm{Np} m_{z}\left(\right.$ units of $\left.\mu_{\mathrm{B}}\right)$ & $1.70(7)$ & $1.66(7)$ & & & 0.0198 \\
$R_{\text {wp }}$ & 0.0212 & 0.0209 & 0.0205 & 0.0201 & 0.0141 \\
$R_{\mathrm{p}}$ & 0.0155 & 0.0152 & 0.0151 & 0.0146 & \\
\hline \hline
\end{tabular}




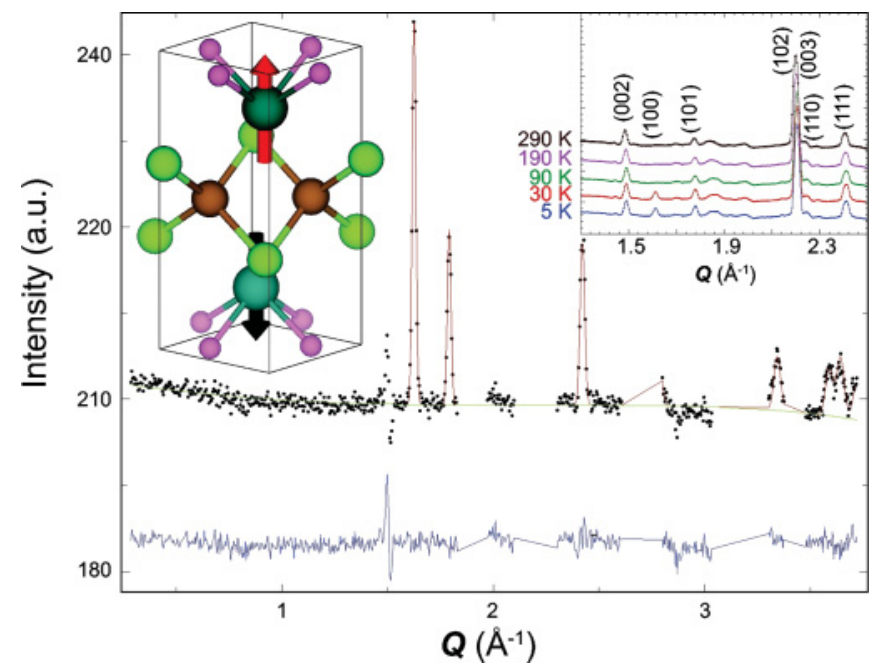

FIG. 4. (Color online) Plot of the Rietveld fit to the difference of the 90 and $30 \mathrm{~K}$ neutron powder diffraction data sets using a $P 4 / n^{\prime} m^{\prime} m^{\prime}$ magnetic model. Portions of the difference spectra where strong nuclear reflections led, due to thermal expansion, to strong up-down features were excluded from the fit. The right inset shows the constant presence of background features at all temperatures in the midmomentum transfer region. The left inset shows the proposed antiferromagnetic structure.

fit was carried out to the difference between the 30 and $90 \mathrm{~K}$ data sets (Fig. 4). A list of reflections, with the measured and calculated structure factors, is shown in Table II. These peaks, indexed using the $P 4 / n^{\prime} m^{\prime} m^{\prime}$ magnetic space group, correspond to the $\mathrm{Np}$ moments being aligned along the $c$ axis, with a ferromagnetic coupling within the basal plane and an antiferromagnetic coupling between the planes (see the left inset in Fig. 4), reminiscent of the low-temperature ordering of the $\mathrm{Sm}$ moments in $\mathrm{SmFeAsO}$. ${ }^{31,32}$

No $\mathrm{Fe}$ moment is observed within the limits of the experiment. At $5 \mathrm{~K}$, the ordered magnetic moment of the $\mathrm{Np}$ ions is $1.70 \pm 0.07 \mu_{\mathrm{B}}$, in excellent agreement with Mössbauer spectroscopy estimations. ${ }^{33}$

TABLE II. Observed, $F_{o}^{2}$, and calculated, $F_{c}^{2}$, structure factors for the magnetic Bragg reflections in the 30-90 K refinement. Structure factors have been corrected for scale and extinction.

\begin{tabular}{lccl}
\hline \hline$h k l$ & $Q\left(\AA^{-1}\right)$ & $F_{o}^{2}$ & \multicolumn{1}{c}{$F_{c}^{2}$} \\
\hline 100 & 1.63 & 0.9312 & 0.9128 \\
101 & 1.80 & 0.2374 & 0.2480 \\
$102^{\mathrm{a}}$ & 2.22 & & 0.04335 \\
111 & 2.43 & 0.4749 & 0.4442 \\
$112^{\mathrm{a}}$ & 2.75 & & 0.4174 \\
$103^{\mathrm{b}}$ & 2.79 & 0.09241 & 0.2043 \\
$113^{\mathrm{a}}$ & 3.23 & & 0.02390 \\
201 & 3.35 & 0.3697 & 0.3177 \\
$104^{\mathrm{a}}$ & 3.43 & & 0.06999 \\
202 & 3.59 & 0.3496 & 0.3294 \\
210 & 3.64 & 0.3925 & 0.4317 \\
$211^{\mathrm{b}}$ & 3.72 & 0.1687 & 0.1349 \\
\hline \hline
\end{tabular}

${ }^{\mathrm{a} E x c l u d e d}$ region.

${ }^{\mathrm{b}}$ Partially excluded region.
It is worth noting that for the parent $R \mathrm{FeAsO} 1111$ compounds, the magnetic rare-earth moments order with the moments varying from 0.83 to 1.55 for $\mathrm{CeFeAsO}$ and $\mathrm{NdFeAsO}$, respectively. ${ }^{34}$ The highest Néel temperature of the rare-earth sublattice in the $R \mathrm{FeAsO} 1111$ series, $T_{\mathrm{N}}(\mathrm{Pr})=$ $14 \mathrm{~K}$ observed for PrFeAsO, ${ }^{35}$ is 4 times lower than $T_{\mathrm{N}}=57 \mathrm{~K}$ estimated for $\mathrm{NpFeAsO}$.

To explore further the electronic and magnetic character of $\mathrm{NpFeAsO}$ we have performed first-principles local-spindensity approximation (LSDA) and LSDA plus Coulomb $U(\operatorname{LSDA}+U)$ calculations. In all calculations we used the crystal-structure parameters determined experimentally at room temperature, assuming the tetragonal $\mathrm{ZrCuSiAs}$ structure. Gaining inspiration from the neutron scattering data, we assumed that the magnetic and crystallographic unit cells coincide. Furthermore, we assumed that the magnetic moment is aligned along the $c$-[001] axis and considered nonmagnetic (NM), ferromagnetic (FM), and AFM arrangements for the $\mathrm{Np}$ moments. There were no magnetic moments set initially on the Fe atoms. In all calculations we assumed stoichiometric $\mathrm{NpFeAsO}$ and did not consider any defects or impurity in the $\mathrm{NpO}_{2}$ phase. The effect of defects and impurities is left for further consideration.

We used an in-house implementation of the full-potential linearized augmented plane wave (FP-LAPW) method. ${ }^{36}$ This FP-LAPW version includes all relativistic effects (scalarrelativistic and spin-orbit coupling) and relativistic implementation of the rotationally invariant LSDA $+U .{ }^{37}$ In the FP-LAPW calculations we set the radii of the atomic spheres to 2.75 a.u. (Np), 2.2 a.u. (Fe, As), and 1.6 a.u. (O). The parameter $R_{N_{p}} K_{\max }=9.625$ determined the basis set size, and the Brillouin zone (BZ) sampling was performed with $405 k$ points. For the neptunium $f$ shell, Slater integrals of $F_{0}=3.00 \mathrm{eV}, F_{2}=7.43 \mathrm{eV}, F_{4}=4.83 \mathrm{eV}$, and $F_{6}=3.53 \mathrm{eV}$ were selected to specify the Coulomb interaction. ${ }^{38}$ They correspond to commonly accepted values for Coulomb $U=$ $3 \mathrm{eV}$ and exchange $J=0.61 \mathrm{eV}$ parameters. The dependence of Np-atom $m_{J}$ on the choice of Coulomb $U$ in a range from 1 to $3 \mathrm{eV}$ was checked, and only small changes in the $m_{J}$ moment value were found.

In LSDA, we found that spin polarization decreases the total energy with respect to the nonmagnetic solution by $0.351 \mathrm{eV}$ per formula unit (f.u.) for the FM solution and by $0.335 \mathrm{eV} /$ f.u. for the AFM solution, suggesting an FM-ordered ground state. However, including the Coulomb $U$ and exchange $J$ in the fully localized limit (FLL) LSDA $+U$ method, we obtain a total energy for the FM solution which is higher by 12 $\mathrm{meV} /$ f.u. than in the AFM solution; on the contrary, if the double-counting correction is made in the around-mean-field limit (AMF), the AFM solution becomes lower in energy than the FM one by $61.2 \mathrm{meV} /$ f.u. Thus, both flavors of LSDA $+U$ yield an AFM-ordered ground state, in agreement with the experiment.

The spin $m_{S}$, orbital $m_{L}$, and total $m_{J}$ magnetic moments for the AFM calculations with LSDA and LSDA $+U$ are shown in Table III. The staggered local magnetic moments, which are due to magnetic polarization of the $\mathrm{Np} f$ shell are formed at the two $\mathrm{Np}$ atoms. Comparison with the experimentally determined moment of $1.7 \mu_{\mathrm{B}}$ shows that LSDA is failing completely to reproduce the results of 
TABLE III. Spin $m_{S}$, orbital $m_{L}$, and total $m_{J}$ magnetic moments (units of $\mu_{\mathrm{B}}$ ) for one of two Np, Fe, As, and O atoms in antiferromagnetic $\mathrm{NpFeAsO}$ resulting from calculations with LSDA and LSDA $+U=3 \mathrm{eV}$ with AMF and FLL double-counting flavors. The last column provides the values obtained from the neutron powder diffraction studies making use of the $g$ value of 0.6 for a free $\mathrm{Np}^{3+}$ ion.

\begin{tabular}{|c|c|c|c|c|c|c|c|c|c|c|c|c|c|}
\hline & \multicolumn{4}{|c|}{ LSDA } & \multicolumn{4}{|c|}{$\mathrm{AMF}$ LSDA $+U$} & \multicolumn{4}{|c|}{ FLL LSDA $+U$} & \multirow{2}{*}{$\begin{array}{c}\text { NPD } \\
\mathrm{Np}\end{array}$} \\
\hline & $\mathrm{Np}$ & $\mathrm{Fe}$ & As & $\mathrm{O}$ & $\mathrm{Np}$ & $\mathrm{Fe}$ & As & $\mathrm{O}$ & $\mathrm{Np}$ & $\mathrm{Fe}$ & As & $\mathrm{O}$ & \\
\hline$m_{S}$ & 2.91 & 0.00 & 0.03 & 0.00 & 1.53 & 0.25 & 0.01 & 0.00 & 2.98 & 0.10 & 0.02 & 0.00 & 2.26 \\
\hline$m_{L}$ & -3.17 & 0.00 & 0.00 & 0.00 & -4.23 & 0.02 & 0.00 & 0.00 & -5.03 & 0.01 & 0.00 & 0.00 & -3.96 \\
\hline$m_{J}$ & -0.25 & 0.00 & 0.03 & 0.00 & -2.70 & 0.27 & 0.01 & 0.00 & -2.05 & 0.11 & 0.02 & 0.00 & -1.7 \\
\hline
\end{tabular}

neutron scattering experiments, while the AMF LSDA $+U$ calculations overestimate the $m_{J}$ moment value. On the other hand, the FLL LSDA $+U$ calculations yield an $m_{J}$ value in reasonable agreement with the experimental data, and the ratio of the orbital to total moment gives a $g$ value of 0.58 , consistent with that for a free $\mathrm{Np}^{3+}$ ion and the value of 0.6 used in the neutron powder diffraction refinements.

It should be noted that when the total energy difference between the FM and the AFM solutions is used to make a "naive" molecular-field theory estimate of the Néel temperature $T_{\mathrm{N}}$, the FLL LSDA $+U$ yields $T_{\mathrm{N}} \sim 47 \mathrm{~K}$, in reasonable agreement with the experimental data. Thus we assume that the LSDA $+U$ model with the FLL double-counting choice gives the most appropriate description of the $\mathrm{NpFeAsO}$.

Although no magnetic polarization on other atoms in the unit cell was initially assumed, the presence of the exchange splitting at the Np atoms leads to induced staggered spin moments on the Fe atoms in a checkerboard AFM arrangement. These are fairly negligible in LSDA but reach $\sim 0.1-0.25 \mu_{\mathrm{B}}$ in LSDA $+U$. Such a moment on iron was not visible in our neutron powder diffraction measurements but cannot be excluded due to the background arising from the sample encapsulation and to the high-absorption cross section of $\mathrm{Np}$.

The total and partial (atom, spin, and orbital resolved) densities of states (DOS) are shown in Fig. 5, calculated with

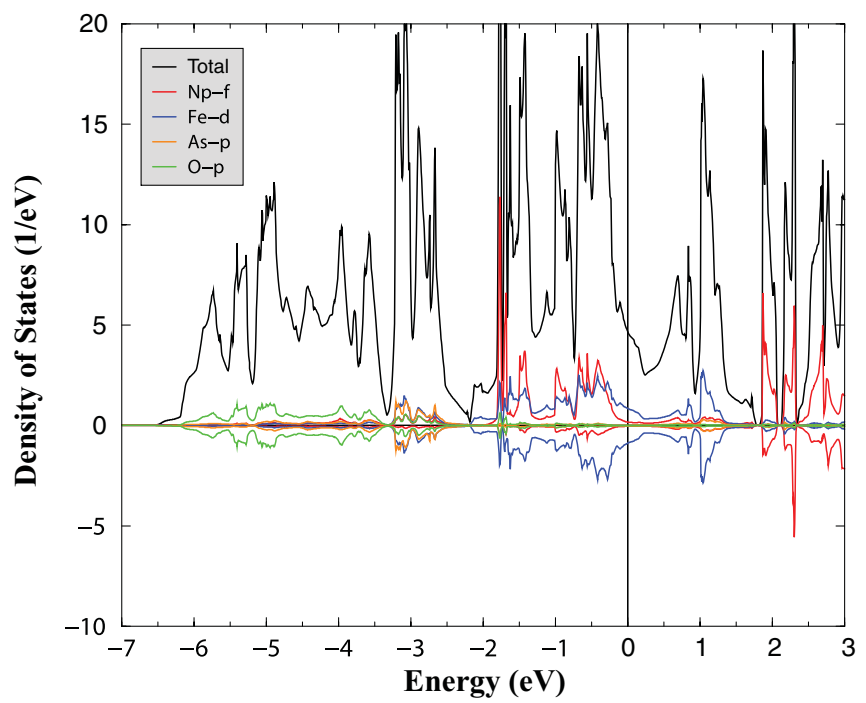

FIG. 5. (Color online) Electronic structure calculations for $\mathrm{NpFeAsO}$ : the total DOS (per unit cell), and atom, spin and orbital resolved DOS as a result of the FLL + LSDA $+\mathrm{U}=3 \mathrm{eV}$ calculations.
FLL LSDA $+U=3 \mathrm{eV}$. The DOS near the Fermi energy $E_{F}$ has mostly $\mathrm{Fe} d$ character, while the As and $\mathrm{O} p$ states are mostly located at the 2-7-eV energy interval below $E_{F}$. The $\mathrm{Np} f$ states are split by the exchange interaction. The resulting DOS at $E_{F}$ of 4.8 states/eV corresponds to the noninteracting value of the Sommerfeld coefficient $\gamma=5.6 \mathrm{~mJ} \mathrm{~mol}^{-1} \mathrm{~K}^{-2}$, which is substantially lower than the experimental value of $75 \mathrm{~mJ} \mathrm{~mol}^{-1} \mathrm{~K}^{-2}$. However, the theoretical value of the Sommerfeld coefficient is expected to increase due to electron mass enhancement caused by dynamical electron interactions and electron-phonon coupling.

The band structure and the Fermi surface (FS) are shown in Fig. 6. The FS consists of five sheets, each of them doubly degenerate. Examination of the band structure shows that sheets FS-1 to FS-3 are holelike and centered at the $\Gamma$ point. Sheets FS-4 and FS-5 are electron-like and centered at the $M$ - $A$ line. Note the fairly two-dimensional character of the FS and the strong resemblance to those previously presented for $R F$ FeAsO. ${ }^{39,40}$ It is seen that most of the states in the vicinity of the FS are located near the $\Gamma-[0,0,0]$ and $M-[\pi, \pi, 0] k$ points in the BZ, suggesting the possibility of an $s \pm$ superconducting pairing mechanism. ${ }^{41}$

A striking feature of $\mathrm{NpFeAsO}$ which makes it different from its rare-earth-based counterparts is the absence of the orthorhombic structural distortion associated with the magnetic ordering. In order to understand this, we performed magnetic anisotropy energy (MAE) calculations, rotating the staggered AFM magnetization from the $c$-axis to the $a$-axis direction. What we found is that the total energy $E_{\text {tot }}$ difference $E_{\mathrm{tot}}(a)-E_{\mathrm{tot}}(c)$ between these two directions of the magnetization is $\approx 30.0 \mathrm{meV} / \mathrm{f}$.u. This means that there is a strong positive uniaxial MAE in $\mathrm{NpFeAsO}$. This MAE keeps the staggered AFM magnetization along the tetragonal $c$ axis and assists in the prevention of any distortion in the $a-b$ plane.

Finally, we turn to the discussion of the negative thermal expansion (NTE). The most recent theory of the Invar effect ${ }^{42}$ is based on the use of a disordered local moment (DLM) approximation. The paramagnetic state in the DLM approximation is treated as a disordered pseudoalloy with equal concentrations of randomly oriented "up" and "down" local moments. It is usually implemented by making use of alloy theory in a coherent-potential approximation. Regrettably, it is not currently possible to use the DLM approximation together with the FP-LAPW basis used in this work.

Therefore, here the LDA + Hubbard-I approximation (HIA), as implemented in Ref. 43, is used to treat the Np-atom local-moment paramagnetic phase. The same values for the Slater integrals for $\mathrm{Np}$ atoms as in LSDA $+U$ were used in 

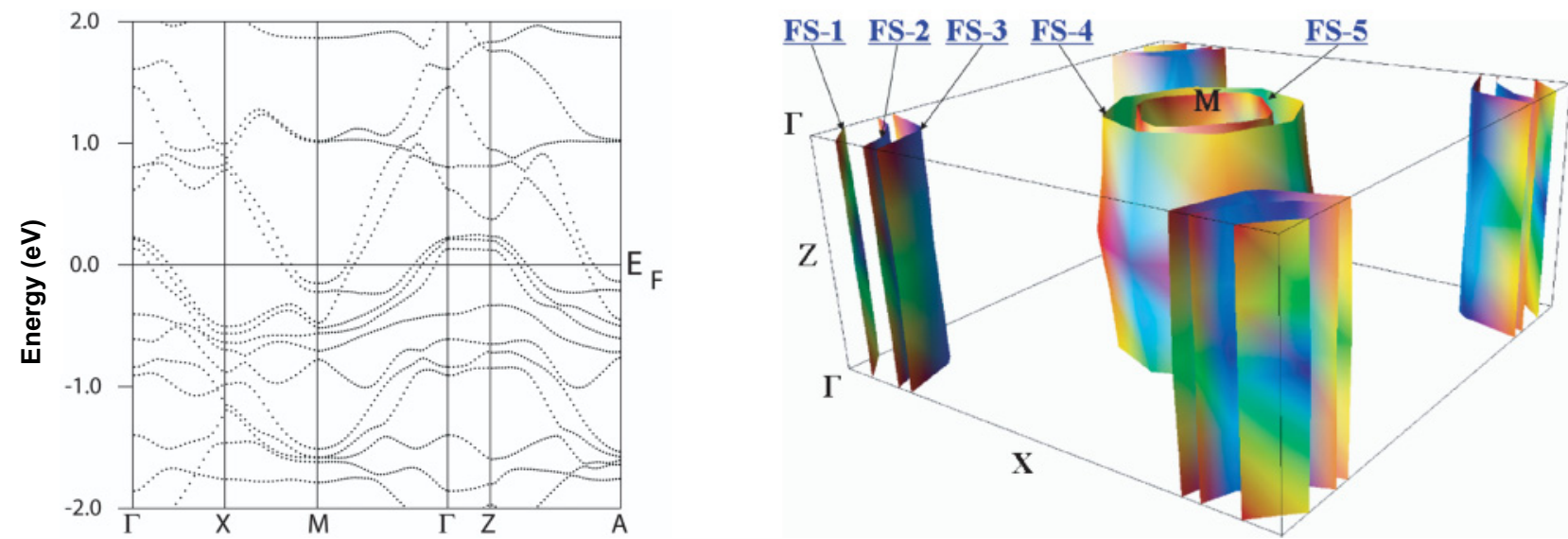

FIG. 6. (Color online) (left) Band structure for NpFeAsO and (right) Fermi surface for NpFeAsO, calculated using the FLL LSDA $+U=$ $3 \mathrm{eV}$ calculations.

the LDA + HIA calculations (which correspond to a Coulomb $U=3 \mathrm{eV}$ and an exchange $J=0.61 \mathrm{eV}$ ), and the inverse temperature $\beta=40 \mathrm{eV}^{-1}$ was chosen. The use of atomic-like $\mathrm{LDA}+\mathrm{HIA}$ is justified for $\mathrm{NpFeAsO}$ at high temperature since the experiments presented above show a well-localized character of the Np-atom $f$ shell above the Néel temperature. Furthermore, the effective paramagnetic moment of $2.84 \mu_{B}$ per $\mathrm{Np}$ atom calculated in LDA + HIA is in very good agreement with the moment of $2.78 \pm 0.06 \mu_{B}$ obtained from susceptibility measurements.

The total energy versus volume was calculated for LDA, LDA + HIA and LSDA $+U$, and the equilibrium volumes $\left(V_{e q}\right)$ obtained using a third-order polynomial fit is shown in Table IV. The $c / a$ ratio and the internal positions of $\mathrm{Np}$ and $\mathrm{As}$ atoms were kept fixed in these calculations. The resulting $V_{e q}$ for LSDA $+U$ is reasonably close (with $3.3 \%$ difference) to the experimental $V_{e q}=124 \AA^{3}$ at $T=0 \mathrm{~K}$, confirming an adequate choice of the computational model for $\mathrm{NpFeAsO}$. However, the calculated $\omega$ of $3.1 \%$, although agreeing in sign, is about 10 times bigger than the experimental value $(\omega=0.2 \%)$.

This quantitative disagreement between theory and experiment is traced to the incorrect description of spin fluctuations on the Fe atoms in LDA + HIA calculations. The Hubbard-I correction is included for the $\mathrm{Np}$ atom only and describes the local-moment paramagnetic phase in $\mathrm{Np}$ sublattices. The Fe-atom sublattice is treated within LDA, with no spin polarization (zero magnetic moment). This approximation is not sufficient for the $\mathrm{Fe}$-atom sublattice in the paramagnetic phase of $\mathrm{NpFeAsO}$. Note that it bears close similarities to what has been found in Ref. 44 for the $\omega$ calculations in ferromagnetic $R \mathrm{Co}_{2}(R=\mathrm{Gd}$, Dy, Er) compounds. The FM state was treated with the so-called open-core approximation

TABLE IV. The equilibrium volume $V_{e q}$ and bulk modulus $\mathrm{B}$ for $\mathrm{NpFeAsO}$ resulting from paramagnetic LDA + HIA and antiferromagnetic LSDA $+U$ calculations.

\begin{tabular}{lrcc}
\hline \hline Method & LDA & LDA + HIA & FLL LSDA $+U$ \\
\hline$V_{e q}\left(\AA^{3}\right)$ & 111.50 & 116.11 & 119.92 \\
$B($ Mbar $)$ & 1.92 & 1.86 & 1.79 \\
\hline \hline
\end{tabular}

for rare-earth atoms, and the DLM approximation was used for the PM phase. The calculated $\omega$ was found to be about 5 times bigger than in the experiment, and no spin polarization on the Co atoms was found in the DLM calculations. By forcing a FM moment on the Co-atom sublattice (applying DLM to the rare-earth sublattice only), the results improved quantitatively, and the value of $\omega$ decreased substantially.

\section{CONCLUSIONS}

In conclusion, we successfully synthesized $\mathrm{NpFeAsO}$, one of the few actinide-based iron-oxypnictide compounds [the other reported oxypnictides containing actinide elements are $\mathrm{U}_{2} \mathrm{Cu}_{2} \mathrm{As}_{3} \mathrm{O}$ (Ref. 45), UCuPO (Refs. 46,47), and $\mathrm{NpCuPO}$ (Ref. 47)]. Although one may expect the physical properties of $\mathrm{NpFeAsO}$ to be similar to the lanthanide 1111 analogs, in reality this system behaves differently. In particular, we do not observe any structural transition, which is in agreement with the fact that neither transport properties (resistivity and the Hall effect) nor specific-heat measurements reveal hightemperature anomalies associated with an in-plane magnetic ordering in the Fe-As layer. We suggest that the lack of the orthorhombic structural distortion is caused by a strong positive uniaxial magnetic anisotropy energy.

Our results reveal that $\mathrm{NpFeAsO}$ exhibits an anomalous thermal expansion which is intimately linked to a giant magnetoexpansion occurring as the system undergoes antiferromagnetic ordering. Negative thermal expansion was observed in $\mathrm{PrFeAsO},{ }^{48}$ and we believe that it is a common feature in the 1111 family with a magnetic trivalent ion. However, the effect in $\mathrm{NpFeAsO}$ is at least 20 times stronger and is clearly associated with the antiferromagnetism of the Np sublattice.

This Invar effect is usually observed in transition-metal disordered alloys [e.g., FeNi, FePt (Ref. 49)] and rare-earth ferromagnets [e.g., $\mathrm{GdCu}_{2}$ (Ref. 50)] and antiferromagnets [e.g., PrFeAsO (Ref. 48)]. It was also found in $\alpha \mathrm{U}$ metal, ${ }^{51}$ and later this effect was explained by the onset of a charge density wave. Even more striking is the observation of a NTE in $\delta \mathrm{Pu},{ }^{52}$ where the absence of magnetic ordering necessitates an alternative mechanism to that based on two spin states with different volumes, as for Invar alloys. ${ }^{53,54}$ Instead, in that 
case it is proposed that the negative thermal expansion is a signature of criticality arising from proximity to a localizationdelocalization transition. ${ }^{55}$ Although uranium and plutonium have rather overshadowed their neighbor neptunium, ${ }^{56}$ here, in reporting the observation of a NTE in $\mathrm{NpFeAsO}$, neptunium will finally take center stage. To the best of our knowledge, $\mathrm{NpFeAsO}$ is the first actinide-based antiferromagnetic material in which negative thermal expansion has been observed. This behavior originates from the magnetic order, but more exotic explanations, e.g., possible criticality at the interface between localized and itinerant electrons, should also be taken into consideration.

\section{ACKNOWLEDGMENTS}

This work has been performed at the Institute of Transuranium Elements within its "Actinide User Laboratory" program, with financial support to users provided by the European Commission. The $\mathrm{Np}$ metal required for the fabrication of the compound was made available through a loan agreement between Lawrence Livermore National Laboratory and ITU in the framework of a collaboration involving Lawrence Livermore National Laboratory, Los Alamos National Laboratory, and the US Department of Energy. The support from Czech Republic Grants No. GACR P204/10/0330 and No. GAAV IAA100100912 is thankfully acknowledged. Work at Princeton University was supported by US DOE Grant No. DE FG02-98ER-45706. T.K. acknowledges the European Commission for financial support in the framework of the "Training and Mobility of Researchers" program. The authors are grateful to everyone who helped with the experiments and preparation of this paper, in particular, F. Kinnart (ITU), A. Hesselschwerdt (ITU), and L. Havela (Charles University, Prague). *tomasz.klimczuk@ec.europa.eu

${ }^{\dagger}$ Present address: Johnson Matthey Technology Centre, Sonning Common, United Kingdom.

${ }^{1}$ J. Zaanen, Nature (London) 457, 546 (2009).

${ }^{2}$ P. M. Grant, Nature (London) 453, 1000 (2008).

${ }^{3}$ S. A. Kivelson and H. Yao, Nature (London) 7, 927 (2008).

${ }^{4}$ M. R. Norman, Physics 1, 21 (2008).

${ }^{5}$ W. E. Pickett, Nat. Phys. 5, 927 (2009).

${ }^{6}$ Y. Kamihara, T. Watanabe, M. Hirano, and H. Hosono, J. Am. Chem. Soc. 130, 3296 (2008).

${ }^{7}$ B. Maroni, L. Malavasi, M. C. Mozzati, M. S. Grandi, A. H. Hill, D. Chermisi, P. Dore, and P. Postorino, Phys. Rev. B 82, 104503 (2010).

${ }^{8}$ R. Caciuffo, G. H. Lander, J. C. Spirlet, J. M. Fournier, and W. F. Kuhs, Solid State Commun. 64, 149 (1987).

${ }^{9}$ D. Mannix, G. H. Lander, J. Rebizant, R. Caciuffo, N. Bernhoeft, E. Lidström, and C. Vettier, Phys. Rev. B 60, 15187 (1999).

${ }^{10}$ D. C. Wallace, Thermodynamics of Crystals (Dover, New York, 1998).

${ }^{11}$ L. Vočadlo, K. S. Knight, G. D. Price, and I. G. Wood, Phys. Chem. Miner. 29, 132 (2002).

${ }^{12}$ P. Dutta, M. S. Seehra, S. Thota, and J. Kumar, J. Phys. Condens. Matter 20, 015218 (2008).

${ }^{13}$ R. Caciuffo, J. A. Paixao, C. Detlefs, M. J. Longfield, P. Santini, N. Bernhoeft, J. Rebizant, and G. H. Lander, J. Phys. Condens. Matter 15, S2287 (2003).

${ }^{14}$ P. Santini, S. Carretta, N. Magnani, G. Amoretti, and R. Caciuffo, Phys. Rev. Lett. 97, 207203 (2006).

${ }^{15}$ N. Magnani, S. Carretta, R. Caciuffo, P. Santini, G. Amoretti, A. Hiess, J. Rebizant, and G. H. Lander, Phys. Rev. B 78, 104425 (2008).

${ }^{16}$ P. Santini, S. Carretta, G. Amoretti, R. Caciuffo, N. Magnani, and G. H. Lander, Rev. Mod. Phys. 81, 807 (2009).

${ }^{17}$ N. H. Andersen, in Crystalline Electric Field and Structural Effects in f-Electron Systems (Plenum, New York, 1980), p. 373.

${ }^{18}$ E. Colineau, P. Javorský, P. Boulet, F. Wastin, J. C. Griveau, J. Rebizant, J. P. Sanchez, and G. R. Stewart, Phys. Rev. B 69, 184411 (2004).
${ }^{19}$ E. H. Putley, Hall Effect and Related Phenomena, Semiconductor Monographs (Butterworths, London, 1960).

${ }^{20}$ H. Kontani, M. Miyazawa, and K. Yamada, J. Phys. Soc. Jpn. 66, 2252 (1997).

${ }^{21}$ N. Nagaosa, J. Sinova, S. Onoda, A. H. MacDonald, and N. P. Ong, Rev. Mod. Phys. 82, 1539 (2010).

${ }^{22}$ A. S. Sefat, M. A. McGuire, B. C. Sales, R. Jin, J. Y. Howe, and D. Mandrus, Phys. Rev. B 77, 174503 (2008).

${ }^{23}$ M. A. McGuire, R. P. Hermann, A. S. Sefat, B. C. Sales, R. Jin, and F. G. D. Mandrus, New J. Phys. 11, 025011 (2009).

${ }^{24}$ S. Suzuki, S. Miyasaka, S. Tajima, T. Kida, and M. Hagiwara, J. Phys. Soc. Jpn. 78, 114712 (2009).

${ }^{25}$ R. H. Liu, G. Wu, T. Wu, D. F. Fang, H. Chen, S. Y. Li, K. Liu, Y. L. Xie, X. F. Wang, R. L. Yang et al., Phys. Rev. Lett. 101, 087001 (2008).

${ }^{26}$ N. Magnani, P. Santini, G. Amoretti, R. Caciuffo, P. Javorský, F. Wastin, J. Rebizant, and G. H. Lander, Phys. B 359-361, 1087 (2005).

${ }^{27}$ T. Klimczuk, J.-C. Griveau, P. Gaczynski, R. Eloirdi, E. Colineau, and R. Caciuffo, J. Phys. Conf. Series 273, 012024 (2011).

${ }^{28}$ A. C. Larson and R. B. Von Dreele, Los Alamos National Laboratory, Tech. Rep. LAUR 86-748, 2004 (unpublished).

${ }^{29}$ B. H. Toby, J. Appl. Crystallogr. 34, 210 (2001).

${ }^{30}$ I. S. Anderson, P. J. Brown, J. M. Carpenter, G. Lander, R. Pynn, J. M. Rowe, O. Schärpf, V. F. Sears, and B. T. M. Willis, International Tables for Crystallography, Vol. C, 3rd ed., edited by E. Prince (Kluwer Academic Publishers, Dordrecht, 2006), Chap. 4.4, pp. 430-487.

${ }^{31}$ S. Nandi, Y. Su, Y. Xiao, S. Price, X. F. Wang, X. H. Chen, J. Herrero-Martín, C. Mazzoli, H. C. Walker, L. Paolasini et al., Phys. Rev. B 84, 054419 (2011).

${ }^{32}$ D. H. Ryan, J. M. Cadogan, C. Ritter, F. Canepa, A. Palenzona, and M. Putti, Phys. Rev. B 80, 220503 (2009).

${ }^{33} \mathrm{P}$. Gaczynski (unpublished).

${ }^{34}$ M. D. Lumsden and A. D. Christianson, J. Phys. Condens. Matter 22, 203203 (2010).

${ }^{35}$ J. Zhao, Q. Huang, C. de la Cruz, J. W. Lynn, M. D. Lumsden, Z. A. Ren, J. Yang, X. Shen, X. Dong, Z. Zhao et al., Phys. Rev. B 78, 132504 (2008). 
${ }^{36}$ A. B. Shick, A. I. Liechtenstein, and W. E. Pickett, Phys. Rev. B 60, 10763 (1999).

${ }^{37}$ A. B. Shick and W. E. Pickett, Phys. Rev. Lett. 86, 300 (2001).

${ }^{38}$ K. Moore and G. van der Laan, Rev. Mod. Phys. 81, 235 (2009).

${ }^{39}$ A. Carrington et al., Phys. C 469, 459 (2009).

${ }^{40}$ D. J. Singh and M.-H. Du, Phys. Rev. Lett. 100, 237003 (2008).

${ }^{41}$ A. Chubukov, Annu. Rev. Condens. Matter Phys. 3, 57 (2012).

${ }^{42}$ S. Khmelevskyi, I. Turek, and P. Mohn, Phys. Rev. Lett. 91, 037201 (2003).

${ }^{43}$ A. B. Shick, J. Kolorenc, A. I. Lichtenstein, and L. Havela, Phys. Rev. B 80, 085106 (2009).

${ }^{44}$ I. Turek, J. Rusz, and M. Divis, J. Magn. Magn. Mater. 290-291, 357 (2005).

${ }^{45}$ D. Kaczorowski, M. Potel, and H. Noël, J. Solid State Chem. 112, 228 (1994).

${ }^{46}$ D. Kaczorowski, J. H. Albering, H. Noël, and W. Jeitschko, J. Alloy Compd. 216, 117 (1994).
${ }^{47}$ D. M. Wells, E. Ringe, D. Kaczorowski, D. Gnida, G. André, R. G. Haire, D. E. Ellis, and J. A. Ibers, Inorg. Chem. 50, 576 (2011).

${ }^{48}$ S. A. J. Kimber et al., Phys. Rev. B 78, 140503 (2008).

${ }^{49}$ E. Wassermann, Ferromagnetic Materials (North-Holland, Amsterdam, 1990), p. 237.

${ }^{50} \mathrm{~A}$. Lindbaum and M. Rotter, Handbook of Magnetic Materials (North-Holland, Amsterdam, 2002), Vol. 14, p. 307.

${ }^{51}$ C. S. Barrett, M. H. Mueller, and R. L. Hitterman, Phys. Rev. 129, 625 (1963).

${ }^{52}$ E. Jette, J. Chem. Phys. 23, 365 (1954).

${ }^{53}$ C. E. Guillaume, in Nobel Lectures, Physics 1901-1921 (Elsevier, Amsterdam, 1967), pp. 444-473.

${ }^{54}$ R. J. Weiss, Proc. Phys. Soc. 82, 281 (1963).

${ }^{55}$ S. Y. Savrasov, G. Kotliar, and E. Abrahams, Nature (London) 410 , 793 (2001).

${ }^{56}$ J. Ibers, Nat. Chem. 2, 996 (2010). 